\title{
Archéopages
}

Archéopages

Archéologie et société

43 | 2016

Médecines

\section{Le médecin face à l'homme. Débat}

Propos recueillis par Catherine Chauveau

Joël Chandelier, Denis Roland et Catherine Chauveau

\section{CpenEdition}

Journals

Édition électronique

URL : https://journals.openedition.org/archeopages/2347

DOI : 10.4000/archeopages.2347

ISSN : 2269-9872

Éditeur

INRAP - Institut national de recherches archéologiques préventives

\section{Édition imprimée}

Date de publication : 1 novembre 2016

Pagination : 80-87

ISSN : 1622-8545

Référence électronique

Joël Chandelier, Denis Roland et Catherine Chauveau, « Le médecin face à I'homme. Débat »,

Archéopages [En ligne], 43 | 2016, mis en ligne le 01 décembre 2018, consulté le 03 juin 2021. URL :

http://journals.openedition.org/archeopages/2347 ; DOI : https://doi.org/10.4000/archeopages.2347

(c) Inrap 


\section{Le médecin face à l'homme}

Si la médecine a acquis un statut à part au sein des sciences,

et si ses praticiens occupent et revendiquent une position sociale

dominante, c'est moins par son pouvoir sur la vie et sur la mort, qu'elle n'a pris que depuis peu, que par ce qui a été son grand but, de l'Antiquité au XIX ${ }^{\mathrm{e}}$ siècle : s'intéresser à ce qui fait l'homme.

\section{Débat Joël Chandelier}

80 enseigne au département d'histoire de l'université Paris 8. Il est membre des équipes de recherche « Histoire des pouvoirs, savoirs et sociétés » (université Paris 8) et « Savoirs et pratiques du Moyen Âge au XIx ${ }^{e}$ siècle » (École pratique des hautes études). Spécialiste d'histoire intellectuelle, d'histoire des sciences et plus particulièrement d'histoire de la médecine, il a publié notamment: Avicenne et la médecine en Italie. Le Canon dans les universités (1200-1350), à paraitre en 2017 aux éditions Champion ; Frontières des savoirs en Italie à lépoque des premières universités (XIII ${ }^{e}-X V^{e}$ siècles), dir. Joël Chandelier et Aurélien Robert, Rome, École française de Rome, 2015 ; «Rhétorique scolastique et enseignement médical (Italie, $\mathrm{XIV}^{\mathrm{e}}$-XV $\mathrm{XV}^{\mathrm{e}}$ siècles) », dans La rhétorique médicale à travers les siècles, éd. Joël Coste, Danielle Jacquart et Jackie Pigeaud, Genève, Droz, 2012, p. 209-228 ; «Expérience, expérimentation et connaissance dans la médecine scolastique italienne du XIV ${ }^{e}$ siècle », dans Expertus sum. L'expérience par les sens dans la philosophie naturelle médiévale, éd. Thomas Bénatouil et Isabelle Draelants, Florence, Sismel, 2011; " Medicine and Philosophy », dans Encyclopedia of Medieval Philosophy, éd. Henrik Lagerlund, Heidelberg, Springer, 2010 p. 745-752.

\section{Denis Roland}

est attaché de conservation du patrimoine au musée de l'école de médecine navale, rattaché au musée national de la Marine. Il est en charge des collections et de la médiation au musée de la Marine et à l'école de médecine navale. Il travaille sur l'histoire de l'arsenal maritime et plus particulièrement sur celle du service de santé à Rochefort. Il a publié notamment : « Les restes humains : questions de médiation. L'exemple de l'école de médecine navale à Rochefort ", dans Techné, n ${ }^{\circ} 44$ à paraître en décembre 2016 ; avec Alain Morgat, «L'hôpital de la Marine de Rochefort, 1788 », dans Revue de la Société française d'histoire des hôpitaux, n¹55, mai 2016 ; Ancienne école de médecine navale. Rochefort, éditions du musée national de la Marine, coll. les Carnets de bord, 2008.
Propos recueillis par Catherine Chauveau. Merci à Christiane Descombin.

Merci à la bibliothèque du musée national de la Marine à Paris pour son accueil. 

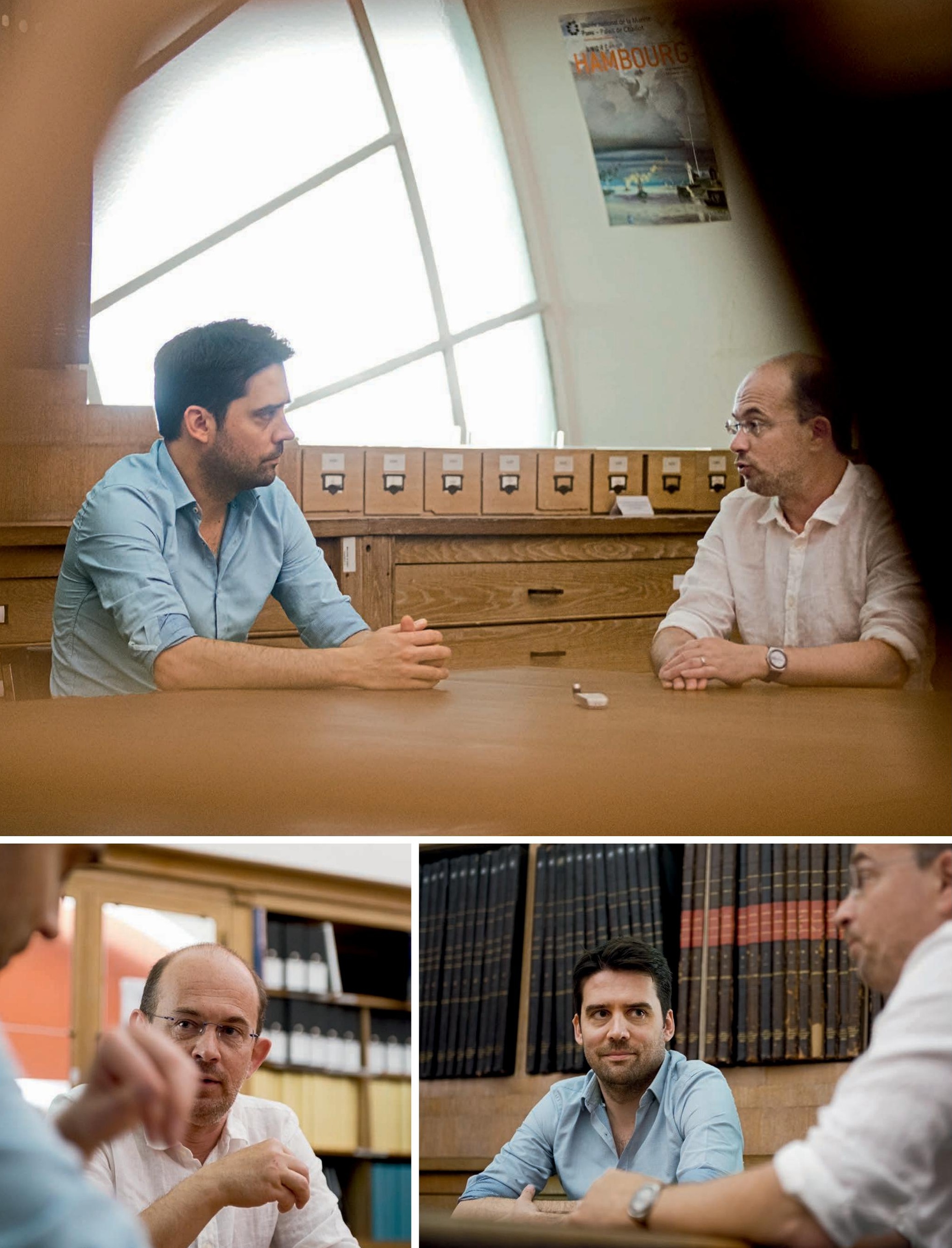
Joël Chandelier S'intéresser à la médecine médiévale, c'est prendre en compte deux aspects particuliers de cette science. Le premier est sa proximité obligée avec la société ; ce n'est pas une science abstraite. Un médecin ne peut se contenter de dire que sa théorie est juste, car elle se heurte obligatoirement à une validation sociale. Ce consentement incontournable de la société est d'autant plus intéressant dans le cas où la théorie est fausse : car il y a des applications qui fonctionnent, malgré la théorie ou à cause d'elle, ou qui ne fonctionnent pas mais sont cependant acceptées socialement. Le second est que la médecine médiévale, et cela reste vrai jusqu'à la fin de l'époque moderne, repose sur une théorie médicale reconnue depuis l'Antiquité et fondamentalement fausse du point de vue scientifique actuel (le système des humeurs notamment). Mais pour autant, c'est une science et on doit la considérer comme telle, pas tant pour la vérité de ce qu'elle dit que pour la méthode utilisée basée sur des observations, sur des expériences, sur des raisonnements, toute une érudition venue de l'Antiquité et qu'il nous faut définir.

Denis Roland Tout à fait ! Peu importe que le savoir et la pratique d'avant soient mieux ou moins bien, ce qui prime est d'avoir cherché et de continuer à le faire. La relativité du savoir scientifique est complètement inscrite dans l'histoire de l'école navale, dès sa création en 1722 , et encore plus fortement au XIX ${ }^{\mathrm{e}}$ siècle. À cette époque, l'école se développe sous la forme qu'on peut visiter aujourd'hui. En premier lieu, une grande bibliothèque, qui s'organise comme les bibliothèques des grandes écoles. Les disciplines traitées dans les livres se retrouvent dans les collections qui en sont l'illustration en trois dimensions. Car la particularité de l'enseignement ici est de n'être pas seulement un enseignement purement livresque; il faut qu'il y ait des échantillons, des objets, des témoins. Au $\mathrm{XVIII}^{\mathrm{e}}$ siècle, les enseignants de l'école s'appellent des démonstrateurs car ils démontrent ce qui est écrit dans le livre, et le donnent à observer aux élèves. La collection, à but pédagogique, donne ce sentiment que le savoir scientifique vient de loin et qu'un scientifique est détenteur d'un état de connaissances en évolution. Avec la très forte spécialisation qui caractérise la science d'aujourd'hui, ce sens de la relativité du savoir se perd beaucoup. On n'apprend plus l'histoire de la médecine dans les universités de médecine, sauf de manière très marginale, et cela influe sur la façon dont le médecin pratique son métier. Dans le musée, on trouve plusieurs collections d'anatomie humaine, évidemment, mais le fait majeur dans ce lieu, comme dans la bibliothèque, est la transversalité des sciences. Être médecin, c'est être un savant au sens XVIII ${ }^{\mathrm{e}}$-XIX ${ }^{\mathrm{e}}$, c'est-à-dire celui qui s'efforce de toujours mieux comprendre le corps et son fonctionnement, mais aussi son environnement.
JC C'est déjà cela qui constitue la figure du médecin médiéval, un savant complet. Le terme pour médecin est physicus, physician en anglais. Et dans les manuscrits, ces termes, comme souvent, sont abrégés et on ne sait jamais si l'on doit lire physicus ou philosophicus, philosophus, parce que les deux se confondent presque. Être médecin, c'est être un presque philosophe puisque c'est s'intéresser à tous les champs de la science. C'est une conception très affirmée dès le milieu du Moyen Âge, à partir du développement des universités au XIII ${ }^{\mathrm{e}}$ siècle, et jusqu'au $\mathrm{XIX}^{\mathrm{e}}$ voire $\mathrm{au} \mathrm{XX}^{\mathrm{e}}$ siècle. Ensuite, la spécialisation devient prédominante et c'est sur elle que se déplace l'excellence. Les médecins médiévaux s'intéressent à tant d'autres disciplines que la leur ; celles liées à la médecine, comme la biologie et la physiologie, et d'autres sans rapport direct pour nous, comme les mathématiques et l'astronomie-astrologie. Tout le monde au Moyen Âge est absolument persuadé que les astres, le soleil et la lune, mais aussi les étoiles, ont une influence sur le monde terrestre. Le cours des astres étant étudié et prévisible, peut-on mesurer et anticiper cette influence? Un des grands débats entre savants est de décider si le médecin doit être astrologue-astronome, pour prédire ce qui va se passer et intervenir, avant des épidémies notamment. On considérait qu'une des causes des épidémies était le déplacement des étoiles qui créait des situations dangereuses, des perturbations climatiques. Cela n'a pas de rapport avec l'observation du monde, mais avec la recherche de grandes causes abstraites déterminant la condition humaine. Car à l'époque médiévale, la question que se posaient les médecins, comme l'ensemble de la société, est principalement celle de la nature de l'homme; l'homme étant placé très au-dessus des autres espèces, on ne s'intéresse à elles que sous le rapport de leur utilité pour l'homme. Cette place à part de l'homme tient à des considérations métaphysiques : la grande particularité de l'homme est d'avoir une âme, une âme rationnelle, qui nous permet de réfléchir, d'être sauvé par Dieu. On pense que les animaux ont aussi une âme, mais une âme animale. Et notre âme est liée à notre corps, du moins dans la vie sur terre. C'est un questionnement anthropologique au sens le plus ancien : qu'est-ce que l'homme? C'est parce que ce questionnement apparaît comme le fondement de la médecine que, dans les éloges de cette science aux XIV $\mathrm{XV}^{\mathrm{e}}-\mathrm{XV}^{\mathrm{e}}$ siècles, on la consacre comme la plus belle des sciences. Pour correspondre à cette image, les médecins sont obligés de connaître toutes les sciences, au moins un peu de chacune, sinon ils ne pourront être de bons médecins, des médecins accomplis.

DR Et aujourd'hui, ce sont les philosophes qui viennent nous parler de la nécessaire transversalité des sciences. En vous écoutant, j'ai l'impression que lorsqu'on crée ces écoles de chirurgie de marine, on crée une formation tout à fait 


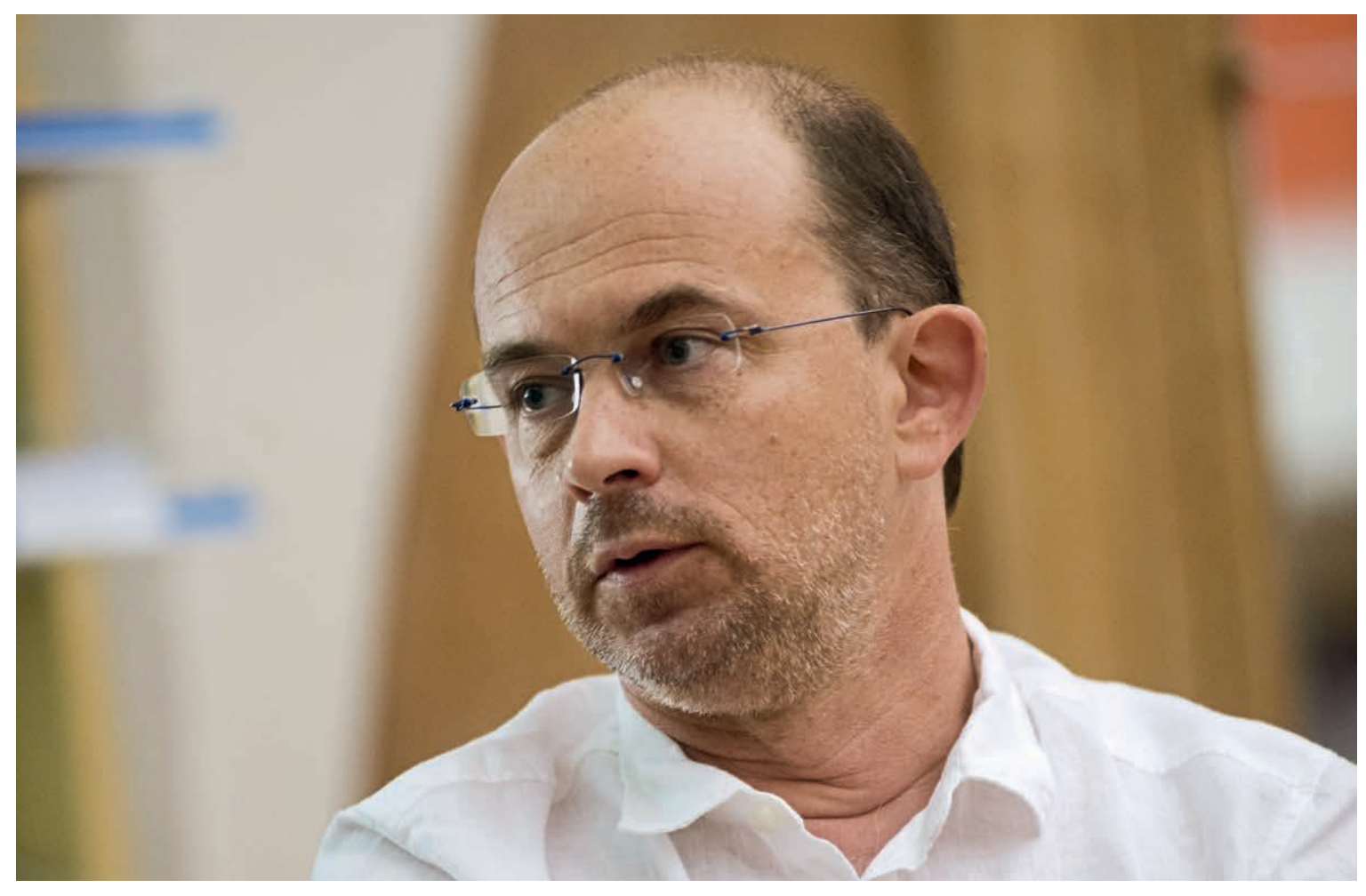

\title{
L'enseignement des officiers de santé reposait sur la transversalité et la relativité du savoir.
}

\author{
Denis Roland
}

anticonformiste. Dans les autres corps d'armée, on forme à la médecine de guerre, qui est déjà particulière. Ces chirurgiens qu'on appelle des officiers de santé ne sont pas des médecins au sens plein du terme. On attend d'eux qu'ils soient efficaces à bord, qu'ils prennent leur place dans l'équipage et qu'ils soient capables d'intervenir dans l'urgence. Et qu'ils n'aillent pas se prendre pour des médecins, car ce sont des manuels. Les officiers de santé me semblent être les héritiers de cette conception de la médecine venue du Moyen Âge, au moment où les médecins s'en détournent pour développer des spécialités. Si la formation qui se met en place à partir de 1722 dans ces écoles de santé de la marine est complètement neuve, c'est qu'on y invente une pédagogie en lien avec la réalité de la situation, c'est-à-dire le fait de voir beaucoup de malades avec tous les problèmes de santé possibles. On apprend à soigner des malades dans les arsenaux en mer en étant réellement confrontés à la maladie. Et à l'époque, c'est une qualité rare pour un futur professionnel de santé ! JC L'enseignement de la médecine à l'université est effectivement avant tout théorique jusqu'au $\mathrm{XVIII}^{\mathrm{e}}$ siècle : il repose sur les livres et sur les grands auteurs. Les médecins ont une claire conscience que ce qu'ils enseignent a une application. Mais l'approche pratique se limite aux leçons d'anatomie, généralisées assez tardivement, dont le but principal est d'appuyer le cours, de produire des images anatomiques. Il s'agit de faire le lien entre ce qu'on a lu dans les livres et la réalité, d'un point de vue purement anatomique, plus rarement pathologique. Mais, dans les faits, il y a toujours un questionnement entre théorie et pratique parce que c'est le cour de la discipline. En ce qui concerne la chirurgie, c'est un peu différent car ces deux professions sont structurellement séparées dans la société médiévale. Au XIV ${ }^{\mathrm{e}}$ siècle, Henri de Mondeville ${ }^{\mathbf{1}}$, un très grand chirurgien de l'époque, plaide auprès du roi de France pour que la chirurgie ne soit plus séparée de la médecine. Selon lui, être un bon chirurgien implique de connaître les causes de ce que l'on opère. Il préconise un enseignement chirurgical, et médical en général, appuyé sur la relation réciproque entre la théorie et la pratique. Ce point de vue n'est pas accepté, et le roi de France maintient une séparation stricte entre les médecins, formés à l'université, et les chirurgiens formés hors université. Cette situation dure jusqu'au $\mathrm{XVII}^{\mathrm{e}}$ siècle et installe la stricte transmission de maître à élève au sein de corporations. C'est ce vide pédagogique qui,
1. Chirurgien et médecin de Philippe le Bel à Montpellier et Paris. Il est l'auteur d'une très portante Chirurgie, sa mort, dans laquelle il s'efforce de donner un statut élevé à la chirurgie, scientifique. 
à l'époque moderne, entraîne la création d'écoles où va éclore l'innovation dans l'enseignement et dans le développement de métiers, parce que l'université était devenue pour certains un cadre trop limité C'est une situation qui cadre avec la création de l'école navale de Rochefort. DR Oui, dans un contexte politique et militaire fort qui est le grand moment de mise en place d'une marine de guerre permanente, après le travail gigantesque de la création des arsenaux voulue par Colbert. Si cette prise en main de la formation par l'État se fait dans le cadre de la Marine, c'est parce que la Marine est confrontée à des problèmes sanitaires effarants. Imaginons ce que peut occasionner la cohabitation de mille hommes campant pendant des mois dans un espace extrêmement confiné avec une promiscuité effarante : il y a des accidents, des épidémies, des problèmes graves liés à la conservation des aliments. Cela entraîne un fort taux de mortalité et la Marine a du mal à constituer des équipages. Avoir des médecins et des chirurgiens à bord est une nécessité stratégique. Les premiers chirurgiens barbiers ont embarqué dans les vaisseaux les plus importants dès 1650 . Mais on se rend vite compte qu'ils sont complètement dépassés. Jean Cochon-Dupuy ${ }^{2}$, le fondateur de l'école de Rochefort, explique leur relative incompétence par leur manque de connaissances notamment anatomiques. Sa première originalité est d'écrire ses cours en français et non en latin. Il dispense un savoir précis et exigeant mais qui se veut pratique et accessible. Le recrutement de l'école à sa création repose sur des conditions très simples : avoir plus de treize ans, savoir lire et écrire, être habile et avoir des mains sans difformités. Pendant tout le XVIII ${ }^{\mathrm{e}}$, cette carrière est un ascenseur social qui fonctionne réellement. Elle est attirante à deux titres (proche de la médecine et dans la marine) et s'ouvre à des gens de condition modeste qui n'auraient pu intégrer aucune de ces professions. Progressivement, ce recrutement se restreint et, à la fin du XVIII ${ }^{e}$ siècle, priorité est donnée aux fils de chirurgiens.

JC Ce recrutement social des chirurgiens barbiers est équivalent au Moyen Âge ; c'est une corporation artisanale en réalité. Les barbiers... font la barbe et d'autres activités qui ne sont pas directement chirurgicales, mais liées au soin global de la personne, en lien avec l'utilisation d'une panoplie d'outils tranchants-coupants. Le fait d'inciser le corps n'est pas un acte anodin. Ils font les saignées, par exemple, qui relèvent du soin et de la prévention. Ce sont donc des professionnels de l'hygiène corporelle, habitués à inciser le corps et certains se spécialisent dans les actes chirurgicaux. Chez les médecins, la plupart le sont de père en fils, comme il y a des généraux de père en fils; rares sont ceux partis de rien et arrivés grâce à l'université. Mais pour entrer à l'université, il faut déjà connaître le latin, ce qui n'est pas à la portée de tout le monde. Dans la haute société, tout le monde a un médecin et les médecins sont souvent riches. La rapacité de cette profession est décrite dans de nombreux textes.

Certaines villes, certaines universités, dépensent des sommes énormes pour faire venir des médecins célèbres. Le chirurgien n'étant en renvanche le plus souvent pas un lettré, pas un clerc, il a un statut social et un salaire moindres. C'est cet écart social que voudront maintenir les médecins qui a, entre autres, empêché un lien plus fort entre chirurgie et médecine. Henri de Mondeville affirme que cette différenciation marquée est complètement injuste, parce que le rôle du médecin se résume à toucher le pouls d'un malade, à regarder son urine, à mettre un nom sur ce dont il souffre et à prescrire un remède et puis... Si le malade meurt, le médecin dira qu'il était déjà trop faible, qu'il n'a pas respecté les proportions, que l'apothicaire s'est trompé dans la préparation, que le barbier a mal fait la saignée... Le médecin, lui, peut toujours s'en sortir. Tandis que le chirurgien, quand on lui apporte quelqu'un qui est encore en vie, si la personne meurt ou s'en sort estropiée, il ne peut pas accuser la nature ou la négligence du malade : pour Mondeville, cela justifie que la chrirugie soit plus noble que la médecine. Le médecin peut garder son prestige, sa réputation, malgré léchec, alors que le chirurgien, à terme, il ne peut que changer de ville, se faire oublier. Au Moyen Âge, il y a ainsi beaucoup de chirurgiens itinérants. C'est sur eux que repose cependant une des trois actions possible du soin, l'actio, l'acte manuel. Les deux autres sont la potio, le médicament sous quelque forme qu'il soit, et la dieta, le régime ou le mode de vie. Mais ces trois actions sont considérées séparément et non en combinaison. La potion est le remède donné à une personne souffrant d'une maladie ; la diète est le régime qu'elle doit suivre pour éviter de tomber malade. Comme on pense que ce sont les excès et les déséquilibres qui rendent malades, qu'ils soient provoqués par la façon de vivre ou des conditions extérieures, la diète préconise un idéal du juste milieu, avec quelques permissions d'excès. Pour ce qui est de l'alcool, par exemple, même un médecin musulman comme Avicenne ${ }^{3}$ dit que s'enivrer à l'excès une fois par mois est utile, car l'alcool renforce le corps et permet d'évacuer les humeurs trop abondante, et donc d'éviter la maladie. Ce juste milieu n'est pas fixé abstraitement mais adapté à chaque individu, selon son sexe, son âge, son tempérament, son activité mais aussi selon la saison ou le climat. Les médecins s'interrogent sur toutes sortes de causes de maladies et en prennent énormément en compte. On a conscience également très tôt de causes psychologiques, du fait, par exemple, qu'on peut tomber malade quand on est mélancolique, terme équivalent au déprimé d'aujourd'hui. Avicenne propose la musique comme remède pour les dépressifs, ou l'activité sexuelle. 


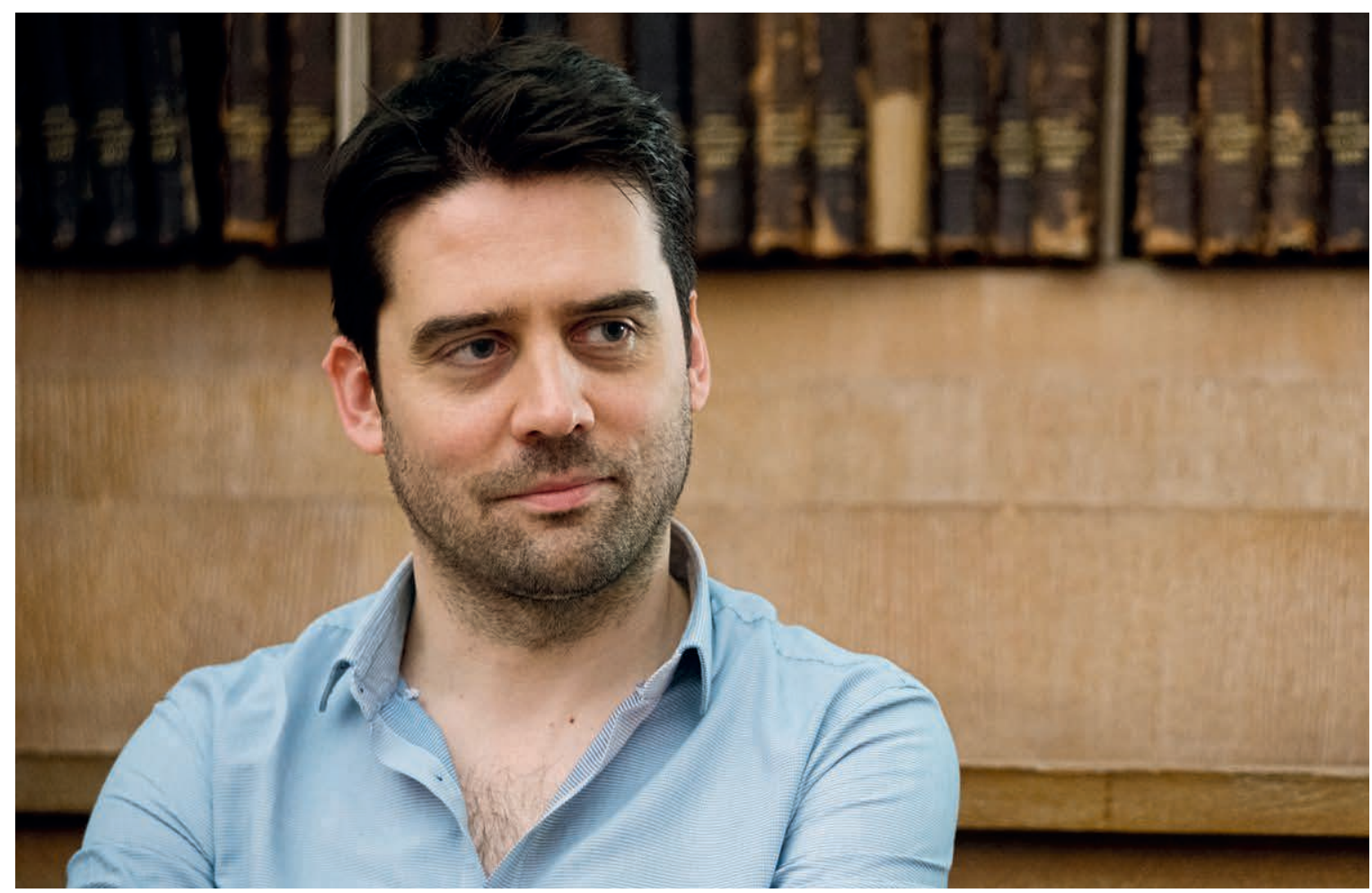

\title{
Jusqu'au XIX ${ }^{\mathrm{e}}$ siècle, la médecine est un questionnement anthropologique.
}

\author{
Joël Chandelier
}

DR Cette interrogation sur les causes est aussi au centre de nos officiers de santé. Mais dans une logique différente, pas du tout en lien avec des réflexions abstraites mais avec des observations pragmatiques. Nous avons dit que l'école est au sein de l'hôpital car il faut côtoyer, visiter les malades et disséquer à tout va. Cette pédagogie qu'on y a inventée développe une culture de l'observation, consignée par écrit, et aboutit à une masse d'enregistrements archivés et transmis : j'ai vu cela, j'ai fait tel geste, il s'est passé cela, je ne sais pas pourquoi mais je le note pour que celui qui arrive après moi le sache. Prenons le cas, fréquent à Rochefort, de la fièvre jaune, ou du paludisme, car la ville est entourée de marais. Un chirurgien dit qu'il y a trop de moustiques et qu'il faut mettre des moustiquaires ; il a vu la relation mais il n'a pas d'explications théoriques. Comme il est en bas de la hiérarchie, et qu'en haut, il y a des médecins qui ont besoin de théorie, sa préconisation n'est pas

4. Navigateur et naturaliste (1754-1803), il commande une expédition composée de deux corvettes, avec une vingtaine de savants à bord, chargée d'explorer les terres australes. suivie. Et on attendra vingt ans, soit des centaines de milliers de morts, pour que cette simple précaution passe dans les mœurs et que soit acceptée la valeur de la seule observation.

JC C'est très intéressant, parce que les médecins du Moyen Âge aussi publient leurs observations mais sous forme de conseils, avec toujours un discours autour. Mais la présentation n'est pas factuelle. Elle relève de la rhétorique, c'est une narration raisonnée qui va au-delà d'un argumentaire. Il est hors de question d'évoquer des expériences ratées, qui pourraient, si elles étaient discutées et analysées, se révéler pourtant très éclairantes et instructives. Et il n'y a pas cette volonté d'enregistrer le monde et de l'inventorier, si forte chez les chirurgiens de la navale. Je me demande si cela est en lien avec leur qualité de chirurgien ou leur qualité de marin?

DR De l'alliance des deux, sans doute, par le rôle qu'ils ont été amenés à assumer à partir du début du XIX ${ }^{\mathrm{e}}$ siècle. En 180o, lors de la grande expédition scientifique organisée par le Directoire et confiée au commandant Nicolas Baudin ${ }^{\mathbf{4}}$, cela se passe très mal entre l'état-major et l'équipe scientifique formée de civils. Ils n'ont pas du tout les mêmes contraintes, ni les mêmes ambitions et ne vivent pas l'expédition de la même manière. Au retour est prise une décision surprenante : les expéditions à venir (qui coûtent affreusement cher et que seul l'État peut monter) ne seront constituées que de militaires et les savants resteront dans leurs muséums. Dans les expéditions qui ont suivi, la partie cartographie et astronomie est donc assurée par l'état-major, formé à cela à l'école navale, et tout 
le reste, relevant principalement de l'histoire naturelle, est confié à d'autres militaires, dont des officiers de santé. C'est pour cela qu'ils jouent un rôle de premier plan dans l'histoire des sciences au cours de la première moitié du XIX ${ }^{\mathrm{e}}$ siècle. C'est ce qui fait aussi que le lieu de l'école navale est si équilibré entre médecine et sciences naturelles, à un moment où se tiennent les grandes controverses sur l'évolution des espèces.

Les officiers de santé apportent un matériau constamment renouvelé à ce débat, auquel ils ne participent pas vraiment d'ailleurs. Ils vont se trouver en charge d'une mission, sans doute déraisonnable, d'inventaire général du monde en géologie, en botanique, en zoologie et aussi en ethnographie, un domaine qui les concerne et les intéresse particulièrement car ils sont souvent les premiers représentants de la science européenne à rencontrer les hommes de contrées lointaines. C'est ainsi que les chirurgiens de marine se sont intéressés aux médecines des autres, parfois avec fermeture d'esprit, parfois avec ouverture. Dans les collections de nos écoles de médecine navale, il y a des aiguilles d'acupuncture, par exemple. Et les officiers de santé pratiquent l'homéopathie très tôt aussi. Tout savoir induit un pouvoir ; ce pouvoir, très faible au début, grandit jusqu'à la création, sous la Révolution, du Conseil de Santé, qui regroupe des chirurgiens et des médecins de la Marine et forme un cercle de décisions sur l'école et l'hôpital mais aussi sur toute question sanitaire. À partir des années 1870, quand on commence à maîtriser l'asepsie et que les chirurgiens sauvent les vies, ils acquièrent la même aura que les médecins. Ces notables jouent un rôle au sein de l'arsenal, puis de la ville. Ils sont des érudits, membres de sociétés savantes de toutes sortes. Les officiers de santé sont des fanatiques d'archéologie, par exemple.

JC Quelles sont les préoccupations relevant du domaine médical des officiers de santé et quels moyens ont-ils à disposition?

DR Ils sont obsédés par la stagnation de l'air pas seulement à bord, mais aussi dans l'hôpital. Dans les bateaux, il faut que l'extérieur soit magnifique, mais à l'intérieur, cela peut être épouvantable. C'est inimaginable de savoir ce que peut être un fond de cale après quatre mois en mer. Les animaux à bord ne restent pas très longtemps car ils sont là pour être mangés mais, en attendant, il faut gérer leurs déjections. Dans ces sociétés où l'on a plus de tolérance aux fortes odeurs qu'aujourd'hui, les cales de bateaux sont perçues comme l'un des endroits les plus puants... Et quand on est sous la ligne de flottaison, on ne peut pas ouvrir la fenêtre. On invente toutes sortes d'appareils à aérer, à fumiger. Même chose dans l'hôpital. Il est conçu, en 1788, avec la préoccupation d'évacuer le souffle des miasmes à l'aide d'un ventilateur central. L'ensemble se compose de plusieurs pavillons séparés, pour limiter des contacts avec l'air vicié, ayant de très grandes hauteurs sous plafond pour favoriser la circulation de l'air. Les hôpitaux de la marine, en général, sont équipés de grands soufflets, de machineries complexes. Ces machines d'aération étant jugées insuffisantes, au XVIII ${ }^{\mathrm{e}}$ et encore plus au XIX ${ }^{\mathrm{e}}$ siècle, la discipline prend de l'importance et se forme l'hygiène navale, un sujet qui s'enseigne. L'idée est d'éviter plus que de remédier. Les questions de l'alimentation sont aussi primordiales. Et l'une des grandes obsessions est le scorbut. Celui qui trouve comment l'éviter ou le guérir gagne la guerre, les autres la perdent, faute de combattants! Pourquoi a-t-on mis tant de temps à comprendre l'origine du scorbut ? Parce l'idée d'une maladie carentielle n'apparaissait pas très concevable. Les médecins, dans la nécessité d'une explication théorique, avaient plutôt tendance à penser que l'on est malade quand on a quelque chose en trop. Les Anglais vont être les premiers, au milieu du XVIII ${ }^{\mathrm{e}}$ siècle, à utiliser le citron, dont les marins ne veulent pas sauf si on le mélange au rhum, et des fruits et légumes frais. Un des objectifs des voyages de Cook $\mathbf{k}^{\mathbf{5}}$ est de porter le triomphe de la médecine venant à bout du scorbut. Il attribue cela à des causes qu'on sait n'être pas tout à fait justes aujourd'hui. Les médecins français sont restés sceptiques sur ce bienfait de fruits et légumes et ont préféré des substances acides, le vinaigre par exemple, qui leur semblaient plus proches de théories médicales.

JC Les historiens de la médecine ne s'intéressent pas assez à cette question des remèdes, des produits ; des matériels aussi d'ailleurs, et l'échange avec les archéologues n'est pas suffisamment développé. La cause en est que, d'une façon générale pour la période médiévale, le rapport entre la science livresque et l'usage pratique est très difficile à faire en grande partie à cause des sources que l'on n'a pas, ou de la façon de ne pas pouvoir les interpréter. Des traités de chirurgie de l'Andalousie musulmane des $\mathrm{X}^{\mathrm{e}}$ et $\mathrm{XI}^{\mathrm{e}}$ siècles sont pleins d'images de toutes sortes de scalpels, avec des formes très précises, et la mention de leurs indications. D'un autre côté, on a quelques corpus d'outils de chirurgie médiévaux, souvent plus tardifs. Mais peut-on établir un lien réel entre les deux? Faisait-on les outils en fonction de ces textes-là ou bien les textes décrivent-ils des outils anciens, encore utilisés ? Ces traités arabes ont fait l'objet de plusieurs copies occidentales : outre la question de la traduction des textes, il y a celle de la reproduction des images. Il y a de fortes divergences de formes, soit que les artistes occidentaux ne voient pas trop la différence entre les images ou l'intérêt de leur réalisme, soit qu'ils aient pris d'autres modèles. D'où que viennent les sources des traités d'outils de chirurgiens médiévaux, ils ne sont ni nombreux ni explicites, au contraire de ceux sur les descriptions de pathologies. Et les artisans fabricants n'écrivent pas de traités. Quant à 
l'iconographie, la plupart du temps son but est de représenter symboliquement l'acte médical. On n'est pas dans la recherche de la précision et de la réalité technique. À la fin du Moyen Âge, les choses commencent toutefois à changer. Un des traités de John Arderne ${ }^{6}$ porte sur le soin de la fistule anale, et comporte des images très précises représentant les outils et les gestes à faire pour les utiliser: cette fois, on a une volonté de transmettre un savoir technique précis. C'est une évolution fondamentale, car en général, à la fin du Moyen Âge, apparaissent des traités décrivant des outils dont l'usage est purement médical. L'invention et la fabrication de ces outils spécifiques sont une question importante qui mérite d'être plus étudiée.

DR La marine étant un cadre très codifié, en tout cas aux XVII ${ }^{\mathrm{e}}$ et XVIII ${ }^{\mathrm{e}}$ siècles, les contenus des coffres aux chirurgiens et d'apothicaires sont listés par écrit, et plusieurs de ces coffres ont été retrouvés en archéologie subaquatique. Il y a quelques années, j'ai été contacté par un archéologue qui avait trouvé un ensemble appartenant au chirurgien du bord : des instruments et objets divers parmi lesquels des fragments de crâne humain ; il lui avait été facile de comprendre qu'il ne s'agissait pas des restes d'un individu mort à bord. On a fini par interpréter ces fragments comme des ingrédients d'un remède très prisé, la thériaque ${ }^{7}$. Ce n'est qu'une hypothèse. Il faut reconnaître que les instruments retrouvés par les archéologues, et bien que ceux des chirurgiens de la marine moderne soient connus et identifiés, nous laissent parfois dans l'hésitation. Au vu des tâches diverses qui incombaient aux officiers de santé, il peut être difficile de lier certains d'eux à leur activité médicale plutôt qu'à leur activité de naturaliste. Entre l'aiguille à suture et l'aiguille à papillon, la différence est minime. De la même façon, les flacons et autres contenants n'ont pas toujours de forme spécifique en lien avec leur usage. Nos collections ne sont pas toujours très documentées. Ce qui fait que, même sur les périodes relativement récentes, la façon dont tous ces objets sont concrètement utilisés ne nous est pas toujours claire. Pour ce qui touche la vie à bord aux XVIII ${ }^{\mathrm{e}}$ et $\mathrm{XIX}^{\mathrm{e}}$ siècles, on a des textes en pagaille (listes d'équipages et d'équipements, journaux de bord, rapports de fin de campagne des chirurgiens...), mais ils ne rendent pas compte de ces aspects. Pour ce qui est de la création d'outils, on observe qu'il y a un énorme investissement des chirurgiens pour l'élaboration de modèles avec des couteliers, dès la fin du $\mathrm{XVIII}^{\mathrm{e}}$ siècle. Cela est évidemment en lien avec des progrès dans la connaissance du fonctionnement du corps humain et avec la spécialisation croissante de la discipline mais on se demande si ce n'est pas également des formes de prestige car l'instrument porte le nom du chirurgien inventeur ; prestige qui peut rejaillir ensuite sur le coutelier, dans une logique de marque. On a beaucoup de ces instruments à l'école navale de Rochefort, et presque tous sont identiques à ceux utilisés aujourd'hui, sauf dans leurs matériaux. Les chirurgiens actuels s'en étonnent souvent et cela semble montrer qu'ils ont perdu l'histoire de leur profession et que la relativité du savoir scientifique se perd un peu chez les scientifiques.

JC Car la différence entre la médecine ancienne, jusqu'au XIX ${ }^{\mathrm{e}}$, et la médecine actuelle est que la première est une médecine holistique tandis que la seconde a pris un parti mécaniste. Les médecins ont atteint aujourd'hui un niveau d'efficacité très élevé sur le plan des maladies aiguës, urgentes et un haut degré de précision des fonctions distinctes de l'organisme. La contrepartie est la perte de la prise en compte de la globalité et de la singularité de chaque patient. Les médecins actuels sont peu armés lorsqu'ils sont confrontés à des maladies moins dangereuses mais pénibles à vivre, comme les maladies chroniques, des maladies qu'on ne guérit pas vraiment, mais dont on pourrait alléger les symptômes de façon à soulager les malades. Ils ont laissé de côté ce qui fut le grand débat théorique de la médecine ancienne, une réflexion sur tous les aspects de ce qu'est une bonne santé qu'ils remplacent par l'addition d'injonctions sanitaires isolées. Galien ${ }^{\mathbf{8}}$ décrit trois états du corps humain : en bonne santé, malade, ou ni l'un ni l'autre, un état neutre. Il introduit cette notion pour mettre en évidence le fait que le plus souvent, on n'est pas dans un état de santé absolue mais dans une situation intermédiaire. Il s'agit de réfuter explicitement la théorie de la souffrance perpétuelle et de définir la santé comme une sorte de but absolu ; le rôle réel du médecin est alors de maintenir un état le plus proche possible de la santé, mais uniquement dans la mesure du possible, par la prévention, dans la durée. Un des exemples de cet accompagnement dans la durée est le thermalisme, qui a toujours existé. Les archives permettent de lister de très nombreux lieux de cure, répartis sur tout le territoire. Ce sont de petits thermes autour desquels se développe une organisation officialisée et professionnalisée, avec des répercussions économiques importantes. C'était une tradition courante, avec ou sans prescription médicale. Les médecins médiévaux voient bien que s'intégrer dans cette tradition de soin est indispensable pour ne pas la laisser à ceux qu'ils considèrent comme des charlatans, et des traités de thermalisme apparaissent au XIV ${ }^{\mathrm{e}}$ siècle. Pour l'instant, les études sont principalement politico-sociales, économiques, intellectuelles mais il n'y a pas beaucoup d'études archéologiques... En tant qu'historiens de la médecine, on aimerait en avoir plus ! (1307-après 1377) est

auteur de plusieurs

7. Du grec therion

l'Antiquité, cette

préparation fut en effet

contrepoison avant

de devenir une panacée,

aux effets aussi variés

8. Philosophe et médecin

grec (129-216) établ

à Rome, il a écrit de

très nombreux traités

d'anatomie et de théorie

médicale suivis jusqu’à

la Renaissance. 